\title{
Grafting of Polymers onto Carbon Microcoil by Use of Carboxyl Groups on the Surface and Electric Properties of Conductive Composite Prepared from Silicone Rubber with the Polymer-Grafted Carbon Microcoil
}

\author{
Haruo Morohashi, ${ }^{1}$ Yukimune TAKahashi, ${ }^{1}$ Yuji NishidA, ${ }^{1}$ Kazuhiro FuJIKI, ${ }^{2}$ \\ Takeshi Yamauchi, ${ }^{1,3}$ Norio TsuboKawa,,${ }^{3,4, \dagger}$ and Seiji Motouma ${ }^{5}$ \\ ${ }^{1}$ Graduate School of Science and Technology, Niigata University, 8050 Ikarashi 2-no-cho, Niigata 950-2181, Japan \\ ${ }^{2}$ Division of Life and Health Sciences, Joetsu University of Education, 1, Yamayashiki, Joetsu, 943-8521, Japan \\ ${ }^{3}$ Center for Transdisciplinary Research, Niigata University, 8050, Ikarashi 2-nocho, Niigata 950-2181, Japan \\ ${ }^{4}$ Department of Material Science and Technology, Faculty of Engineering, Niigata University, \\ 8050, Ikarashi 2-nocho, Niigata 950-2181, Japan \\ ${ }^{5}$ Faculty of Engineering, Gifu University, 1-1, Yanagido, Gifu 501-1193, Japan
}

(Received December 13, 2006; Accepted February 1, 2007; Published March 12, 2007)

\begin{abstract}
Carboxyl groups were successfully introduced on carbon microcoil (CMC) surface by the treatment with nitric acid. The carboxyl group content increased with increasing reaction time and reached to $1.34 \mathrm{mmol} / \mathrm{g}$ after $10 \mathrm{~h}$. Poly(ethylene glycol) (PEG) was grafted onto the CMC surface by the reaction of carboxyl groups with terminal hydroxyl groups of PEG in the presence of condensing agent such as $N, N^{\prime}$-dicyclohexylcarbodiimide or Lewis acid catalyst such as scandium (III) trifluoromethanesulfonate. Carboxyl groups on CMC surface have ability to initiate the cationic polymerization of vinyl monomers, such as isobutyl vinyl ether and $N$-vinylcarbazole and the corresponding polymers were grafted onto the surface. By grafting of polymers onto the surface, the entanglement of CMC was effectively destroyed and polymer-grafted CMC uniformly dispersed in good solvents for grafted polymer and silicone rubber. The inductance, capacitance, and resistance of conductive composite prepared from polymer-grafted CMC responded to acetone vapor. The inductance of the composite from polymer-grafted CMC increased in acetone vapor, and returned to initial value when it was transferred into air. The capacitance and resistance of the composite decreased in acetone vapor, and returned to initial value when it was transferred into air. [doi:10.1295/polymj.PJ2006193]

KEY WORDS Carbon Microcoil / Surface Grafting / Conductive Composite / Dispersibility / Electric Property / Inductance /
\end{abstract}

Carbon microcoil (CMC) is a new class of carbon fiber with 3D-helical/spiral forms with a coil diameter of $\mu \mathrm{m}$ orders, and a possible candidate for electromagnetic absorber, ${ }^{1}$ hydrogen absorber, ${ }^{2}$ and tactile sensor. ${ }^{3}$

It has been reported that parameters of inductance $L$, capacitance $C$, and resistance $R$ of the bulk CMC increased with their extension and decreased with their contraction. It was found that the arraying of the CMC in the silicone rubber matrix resulted in a significant increase in the tactile sensitivity. ${ }^{3-5}$

However, it is difficult to disperse CMC in various solvents and polymer matrices uniformly because of the unique morphology. We have pointed out that the grafting of polymers onto the surface is effective method for the destruction of aggregation of nanoparticles and entanglement of nanofibers. ${ }^{6-8} \mathrm{We}$ have reported the surface grafting of various polymers onto carbon materials, such as carbon black, graphite, carbon nanotubes, and carbon fibers. ${ }^{6-12}$

For example, we have pointed out that carboxyl groups on carbon materials have an ability to initiate the cationic polymerization of $N$-vinylcarbazole and isobutyl vinyl ether to give the corresponding polymer-grafted carbon materials. ${ }^{11,13,14}$ On the other hand, the grafting of poly(ethylene glycol) (PEG) was successfully grafted onto carbon black surface by the reaction with carboxyl groups with hydroxyl groups of PEG in the presence of $N, N^{\prime}$-dicyclohexylcarbodiimide (DCC) as a condensing agent ${ }^{15}$ or $\mathrm{Sc}(\mathrm{OTf})_{3}$ as Lewis acid catalyst. ${ }^{16}$

In the previous paper, we have reported the grafting of polymers by ligand-exchange reaction of ferrocene moieties of polymers with polycondensed aromatic rings of carbon fiber, ${ }^{17}$ carbon nanofibers, ${ }^{18}$ and CMC. ${ }^{19}$ We have pointed out that the surface grafting by the ligand-exchange reaction between ferrocene moieties of the copolymer and the polycondensed aromatic rings of carbon materials is very effective for the grafting of polymers onto carbon materials having few functional groups.

On the other hand, we have reported that the elec-

${ }^{\dagger}$ To whom correspondence should be addressed (Tel/Fax: +81-25-262-6779, E-mail: ntsuboka@eng.niigata-u.ac.jp). 


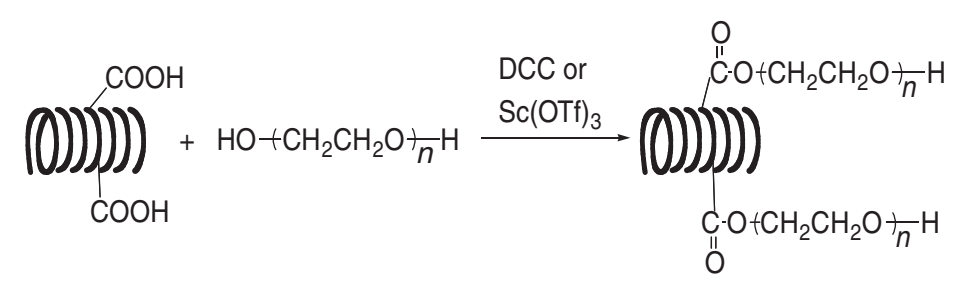

Scheme 1. Grafting of PEG onto CMC by the reaction of carboxyl groups on the surface with PEG in the presence of DCC or $\mathrm{Sc}(\mathrm{OTf})_{3}$.

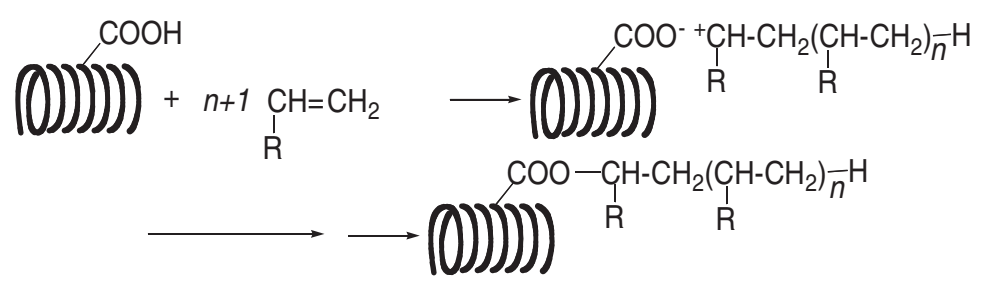

Scheme 2. Grafting of polymers onto CMC during the cationic polymerization initiated by carboxyl groups on CMC.

tric resistance of conductive composite prepared from polymer-grafted carbon black suddenly increase in organic solvent vapor and returned to initial resistance when it was transferred into air. ${ }^{20-23}$

In this paper, to improve the dispersibility of CMC in solvents and polymer matrices, we investigated the grafting of PEG onto CMC surface by the direct condensation of carboxyl groups in the presence of DCC or $\mathrm{Sc}(\mathrm{OTf})_{3}$ as shown in Scheme 1. In addition, the grafting of polymers during the cationic polymerization of initiated by carboxyl groups on CMC was examined as shown in Scheme 2. The dispersibility of polymer-grafted CMC in solvent and silicone rubber will be discussed. The response of inductance $L$, capacitance $C$, and resistance $R$ of conductive composites prepared from silicone rubber and the polymergrafted CMC was investigated.

\section{EXPERIMENTAL}

\section{Materials and Reagents}

Carbon microcoil (CMC) was supplied from CMC Technology Development Co., Ltd. Japan and dried in vacuo at $110^{\circ} \mathrm{C}$ before use. The coil diameter, fiber diameter, and fiber length of the CMC are $1-3 \mu \mathrm{m}$, $0.1 \mu \mathrm{m}$, and $90-150 \mu \mathrm{m}$, respectively. The bulk density of CMC is $1.72 \mathrm{~g} / \mathrm{cm}^{3}$. The CMC was used without further purification.

Isobutyl vinyl ether (IBVE) was refluxed over sodium and distilled before use. $N$-Vinylcarbazole (NVC) was recrystallized from $n$-hexane before use. Toluene and tetrahydrofuran was refluxed over sodium and distilled.

$N, N^{\prime}$-Dicyclohexylcarbodiimide (DCC) and scandium (III) trifluoromethanesulfonate, $\mathrm{Sc}(\mathrm{OTf})_{3}$, were obtained from Aldrich Chemical Co. and used without further purification. Nitric acid (60-61\%) was obtained from Kanto Chemical Ind. Co. and used without further purification.

\section{Poly(ethylene glycol) and Silicone Rubber}

Poly(ethylene glycol), PEG, was obtained from Aldrich Chemical Co. and dried at above melting point under vacuum. The molecular weight of PEGs was $6 \times 10^{2}, 1.5 \times 10^{3}, 3.4 \times 10^{3}, 8.0 \times 10^{3}, 1.0 \times 10^{4}$, and $2.0 \times 10^{4}$.

Silicone rubber (hydroxyl-capped poly(dimethylsiloxane)) used was obtained from Shin-Etsu Chemical Co., Ltd. Japan. The poly(dimethylsiloxane) was cured by silicate in the presence of tin catalyst at room temperature.

\section{Introduction of Carboxyl Groups onto CMC Surface}

Into a flask, $1.0 \mathrm{~g}$ of $\mathrm{CMC}$ and $100 \mathrm{~mL}$ of nitric acid was charged and the reaction mixture was refluxed under stirring slowly with a magnetic stirrer. The stirring rate was controlled below $140 \mathrm{rpm}$ for the prevention of broken of CMC. After the reaction, the resulting $\mathrm{CMC}$ was filtered off by a micro filter and washed with distilled water repeatedly until filtrate became neutral. The treated CMC was dried at $50^{\circ} \mathrm{C}$ in vacuum. The $\mathrm{CMC}$ having $\mathrm{COOH}$ groups was abbreviated as $\mathrm{CMC}-\mathrm{COOH}$.

\section{Determination of Carboxyl Group Content of CMC}

The carboxyl group content of CMC was determined by back titration. ${ }^{24} \mathrm{~A}$ typical example was as follows. Into a flask, $0.03 \mathrm{~g}$ of CMC and $50.0 \mathrm{~mL}$ of $0.10 \mathrm{~mol} / \mathrm{L}$ sodium carbonate aqueous solution were charged and the mixture was stirred at room temperature. After $6 \mathrm{~h}, 20.0 \mathrm{~mL}$ of the supernatant solution was pipetted off and treated with $20.0 \mathrm{~mL}$ of 0.10 
mol/L $\mathrm{HCl}$ aqueous solution. To remove dissolved carbon dioxide in the solution, the solution was refluxed for $30 \mathrm{~min}$ and then the solution was titrated with $0.05 \mathrm{~mol} / \mathrm{L} \mathrm{NaOH}$ aqueous solution using phenolphthalein as an indicator.

\section{Grafting Reaction of Carboxyl Groups on CMC with PEGs}

Into a flask, $0.04 \mathrm{~g}$ of CMC-COOH, $0.11 \mathrm{mmol}$ of PEG, $0.05 \mathrm{mmol}$ of DCC (or Sc(OTf) $)_{3}$ ), and $20.0 \mathrm{~mL}$ of THF were charged and the reaction mixture was stirred slowly with a magnetic stirrer under nitrogen at $60^{\circ} \mathrm{C}$. After the reaction, the resulting $\mathrm{CMC}$ was filtered off by a micro filter and washed with methanol repeatedly until no more PEG could be detected in the filtrate. The PEG-grafted CMC was dried at $50{ }^{\circ} \mathrm{C}$ in vacuum.

\section{Cationic Polymerization of IBVE Initiated by $C M C$ - $\mathrm{COOH}$}

Into a polymerization tube, $0.04 \mathrm{~g}$ of $\mathrm{CMC}-\mathrm{COOH}$ and $15.0 \mathrm{~mL}$ of IBVE were charged and the reaction mixture was stirred slowly with a magnetic stirrer under nitrogen at $25^{\circ} \mathrm{C}$. After the polymerization, the reaction product was poured into large excess of methanol to precipitate polymer-grafted CMC. The precipitate was filtered off by a micro filter and washed with methanol. The polymer-grafted CMC was dried at $50{ }^{\circ} \mathrm{C}$ in vacuum.

\section{Cationic Polymerization of NVC Initiated by CMC- $\mathrm{COOH}$}

Into a polymerization tube, $0.03 \mathrm{~g}$ of $\mathrm{CMC}-\mathrm{COOH}$, $3.0 \mathrm{~g}$ of $\mathrm{NVC}$, and $10.0 \mathrm{~mL}$ of toluene were charged and the reaction mixture was stirred slowly with a magnetic stirrer under dry nitrogen at $80^{\circ} \mathrm{C}$. The procedure after the polymerization was carried out as same manner as mentioned above.

\section{Determination of Grafting}

To remove ungrafted polymer, the reaction product was dispersed in good solvent of grafted polymer and the dispersion was filtered and washed with good solvent repeatedly until no more polymer could be detected in the filtrate. The percentage of polymer grafting was calculated by the following equation.

$$
\text { Grafting }(\%)=(A / B) \times 100,
$$

where $A$ is polymer grafted $(\mathrm{g})$ and $B$ is CMC used (g). The amount of polymer grafted onto the CMC surface was determined by weight loss when the polymer-grafted $\mathrm{CMC}$ was heated from room temperature to $500^{\circ} \mathrm{C}$ under nitrogen at $10^{\circ} \mathrm{C} / \mathrm{min}$ by using a thermal analyzer.

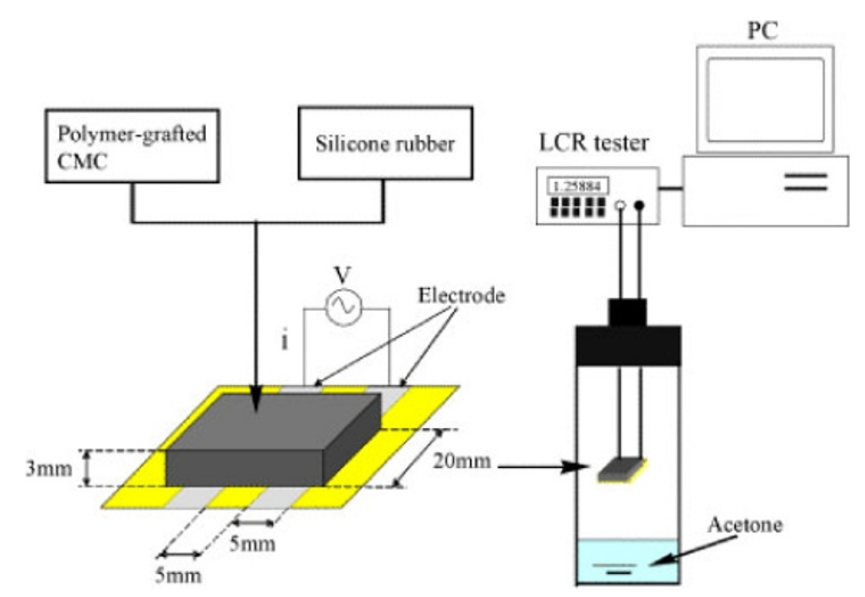

Figure 1. Apparatus for the measurement of inductance, capacitance, and resistance of conductive composite prepared from polymer-grafted $\mathrm{CMC}$ and silicone rubber.

\section{Preparation of Conductive Composite of Silicone} Rubber with Polymer-Grafted CMC

The preparation of conductive composite from polymer-grafted CMC and silicone rubber was carried out as follows. Into a disposable cup, polymer-grafted $\mathrm{CMC}$, poly(dimethylsiloxane) and curing agent were charged. Then, the content of the cup was mixed at room temperature using a hybrid-deforming mixer (Thinky Co., Japan; AR-100) to produce uniform slurry of the composite (the content of CMC was $5 \mathrm{wt} \%$ ). The resulting slurry of the composite was poured into a mold, which has a dimension of $20 \times 20 \times 3 \mathrm{~mm}$. After the slurry of the composite was cured in air at $50^{\circ} \mathrm{C}$ for $1 \mathrm{~h}$, the resulting composite was removed from the mold.

Determination of Inductance L, Capacitance C, and Resistance $R$ of the Conductive Composite

The response of inductance $L$, capacitance $C$, and resistance $R$ of the composite prepared from silicone rubber and polymer-grafted CMC against to acetone vapor was measured as follows. The conductive composite was put on an electrode (distance between the electrodes and width of the electrode were $5 \mathrm{~mm}$ ), which was made of stainless steel placed on a ceramic plate as shown in Figure 1. The composite put on the electrode was transferred into a glass tube containing acetone at the bottom (the distance between the composite and acetone surface was $70 \mathrm{~mm}$ ). Inductance, capacitance, and resistance were measured at the frequency range of $100 \mathrm{kHz}$ with the voltage of $0.1 \mathrm{~V}$ using a LCR tester (LCR HiTESTER 3522-50: HIOKI E. E. Co., Japan) every 5 min in air and then in saturated acetone vapor at $25^{\circ} \mathrm{C}$. The electrode was connected directly to a LCR tester via a four-terminal probe, and the inductance, capacitance, and resistance were read by a personal computer. 


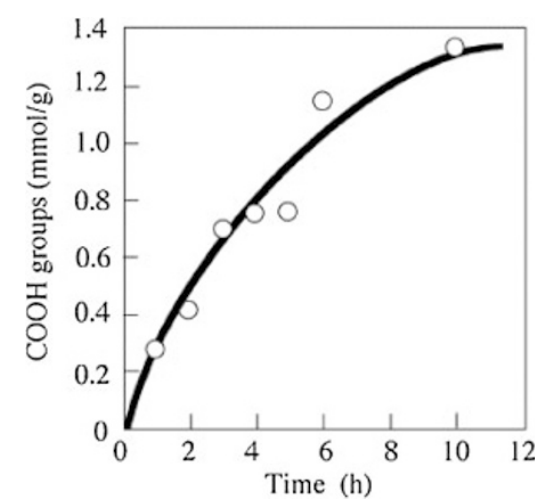

Figure 2. Relationship between reaction time and carboxyl groups introduced onto CMC surface by the treatment with $\mathrm{HNO}_{3}$. CMC, $1.0 \mathrm{~g}$; conc. $\mathrm{HNO}_{3} ; 100 \mathrm{~mL}$; reflux temperature.

\section{RESULTS AND DISCUSSION}

\section{Introduction of Carboxyl Groups onto CMC Surface} by the Treatment with $\mathrm{HNO}_{3}$

CMC scarcely has carboxyl groups on the surface. It is well known that carboxyl groups can be introduced onto carbon materials, such as carbon black, carbon fiber, vapor grown carbon fiber, and carbon nanotube. ${ }^{7,8}$ Therefore, we tried the introduction of carboxyl groups onto CMC by the treatment with $\mathrm{HNO}_{3}$. Figure 2 shows the relationship between reaction time and carboxyl group content on CMC.

It was found that carboxyl group content increased with reaction time and reached to $1.34 \mathrm{mmol} / \mathrm{g}$ after $10 \mathrm{~h}$ at refluxed temperature. The value was much larger than those introduced onto other carbon materials by the reaction with $\mathrm{HNO}_{3}$. Figure 2 also shows that the content of carboxyl groups on CMC can be controlled by the reaction time.

\section{Grafting of PEG onto CMC Surface}

We have reported that hydrophilic PEGs were readily grafted onto carbon black and vapor grown carbon fiber surfaces by the reaction of the surface carboxyl groups in the presence of condensing agent, such as DCC $^{15}$ and $\mathrm{Sc}(\mathrm{OTf})_{3} .{ }^{16}$ Therefore, the grafting of PEG onto CMC by this manner was examined.

Figure 3 shows the relationship between PEG grafting and reaction time. It was found that PEG grafting increased with increasing reaction time and reached to $16.6 \%$ after $24 \mathrm{~h}$. On the contrary, no grafting of PEG onto CMC was observed by the reaction of untreated CMC with PEG even in the presence of DCC as shown in Figure 3 (gray circle).

In addition, $\mathrm{PEG}$ grafting onto $\mathrm{CMC}-\mathrm{COOH}$ with higher carboxyl content $(1.34 \mathrm{mmol} / \mathrm{g})$ was higher than that with lower carboxyl content $(0.76 \mathrm{mmol} /$ g). But carboxyl groups of the former reacted with

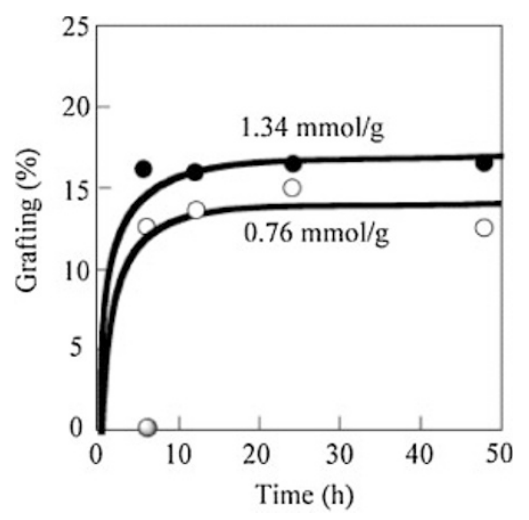

Figure 3. Grafting reaction of $\mathrm{PEG}$ with $\mathrm{CMC}-\mathrm{COOH}$ (open and filled circle) and untreated $\mathrm{CMC}$ (gray circle) in the presence of DCC. CMC-COOH $(\mathrm{COOH}=1.34 \mathrm{mmol} / \mathrm{g}), 0.04 \mathrm{~g}$; PEG $\left(M_{\mathrm{n}}=3400\right), 0.11 \mathrm{mmol}$; DCC, $0.05 \mathrm{mmol}$; THF, $20.0 \mathrm{~mL} ; 60^{\circ} \mathrm{C}$; $6 \mathrm{~h}$.

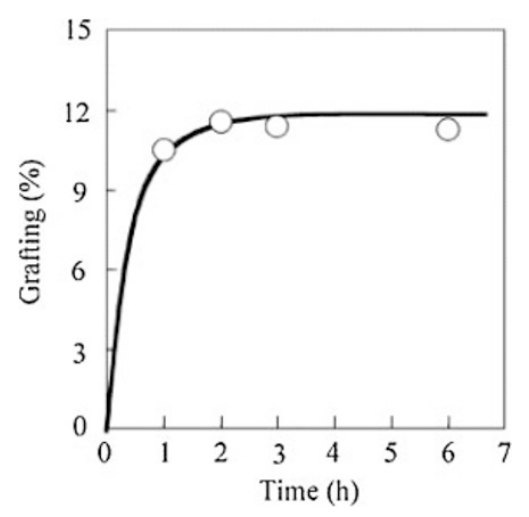

Figure 4. Grafting reaction of $\mathrm{PEG}$ with $\mathrm{CMC}-\mathrm{COOH}$ in the presence of $\mathrm{Sc}(\mathrm{OTf})_{3}$. CMC-COOH $(\mathrm{COOH}=1.34 \mathrm{mmol} / \mathrm{g})$, $0.04 \mathrm{~g} ; \quad$ PEG $\left(M_{\mathrm{n}}=3400\right), 0.11 \mathrm{mmol} ; \mathrm{Sc}(\mathrm{OTf})_{3}, 0.05 \mathrm{mmol}$; THF, $20.0 \mathrm{~mL} ; 60^{\circ} \mathrm{C}$.

PEG was lower than those of the latter. The result indicates that carboxyl groups on CMC used for the grafting reaction decreased with increasing carboxyl group density on CMC surface, because of steric hindrance.

Figure 4 shows the results of the grafting reaction of CMC-COOH with PEG in the presence of $\mathrm{Sc}(\mathrm{OTf})_{3}$ as catalyst. The grafting of PEG onto CMC-COOH was also proceeded in the presence of $\mathrm{Sc}(\mathrm{OTf})_{3}$. The reaction rate in the presence of $\mathrm{Sc}(\mathrm{OTf})_{3}$ was higher than that in the presence of DCC. When $\mathrm{Sc}(\mathrm{OTf})_{3}$ was used as catalyst, $\mathrm{Sc}(\mathrm{OTf})_{3}$ was easily recovered from reaction solvent and can be reused.

\section{Effect of Molecular Weight of PEG on the Grafting onto $C M C$}

Table I shows the effect of molecular weight of PEG on the grafting of $\mathrm{CMC}-\mathrm{COOH}$. As shown in Table I, the percentage of PEG grafting tends to 
Table I. Effect of molecular weight on the grafting of PEG onto CMC-COOH $(1.34 \mathrm{mmol} / \mathrm{g})$ in the presence of DCC

\begin{tabular}{rccc}
\hline \multicolumn{1}{c}{$M_{\mathrm{n}}$} & Grafting $(\%)$ & Grafted PEG $(\mu \mathrm{mol} / \mathrm{g})$ & $R(\%)$ \\
\hline 600 & 14.2 & 237 & 17.6 \\
1500 & 17.4 & 116 & 8.5 \\
3400 & 18.5 & 54.4 & 4.1 \\
8000 & 18.0 & 22.5 & 1.7 \\
10000 & 18.7 & 18.7 & 1.4 \\
20000 & 18.6 & 9.3 & 0.7 \\
\hline \multirow{4}{*}{$R(\%)=$} & Grafted PEG $(\mathrm{mmol} / \mathrm{g})$ \\
& CMC-COOH $(\mathrm{mmol} / \mathrm{g})$
\end{tabular}

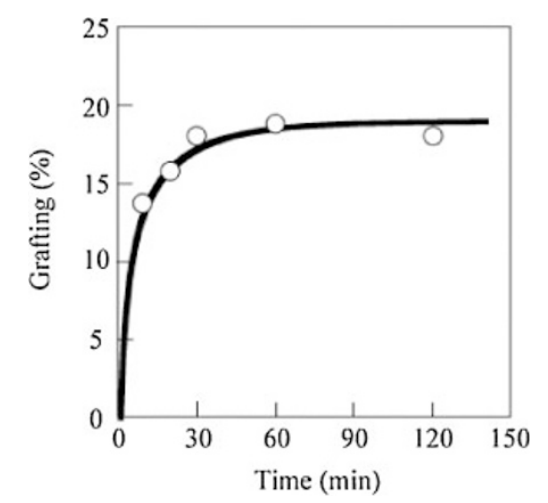

Figure 5. Grafting of polyIBVE onto CMC during the cationic polymerization initiated by $\mathrm{CMC}-\mathrm{COOH}$. $\mathrm{CMC}-\mathrm{COOH}$ $(\mathrm{COOH}=1.34 \mathrm{mmol} / \mathrm{g}), 0.04 \mathrm{~g}$; IBVE, $15.0 \mathrm{~mL} ; 25^{\circ} \mathrm{C}$.

increase with increasing molecular weight of PEG. But the grafted chains on the surface and percentage of carboxyl groups used for the grafting $(R)$ decreased with increasing molecular weight of PEG. This indicates that steric hindrance increased with increasing molecular weight of PEG. The same tendency was observed in the grafting reaction of functional groups on carbon black with functional polymers. ${ }^{15}$

\section{Cationic Polymerization Initiated by $\mathrm{CMC}-\mathrm{COOH}$}

We have reported that carboxyl groups on carbon materials have strong acidity in comparison with aromatic carboxylic acids and have an ability to initiate the cationic polymerization of vinyl monomers. ${ }^{13,14}$ During the polymerization, a part of polymer was grafted onto the surface. Therefore, the initiating activity of $\mathrm{CMC}-\mathrm{COOH}$ for the cationic polymerization was examined.

It was found that the cationic polymerization of IBVE was successfully initiated even at $25^{\circ} \mathrm{C}$. Untreated $\mathrm{CMC}$ has no ability to initiate the cationic polymerization of IBVE. Figure 5 shows percentage of polyIBVE grafting during the cationic polymerization. It was found that polyIBVE grafting increased with progress of the polymerization and reached to $18.5 \%$ after $60 \mathrm{~min}$.

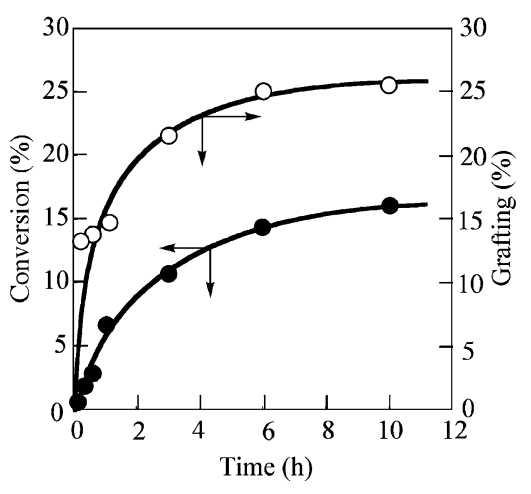

Figure 6. Grafting of polyNVC onto $\mathrm{CMC}$ during the cationic polymerization initiated by CMC-COOH. $\mathrm{CMC}-\mathrm{COOH}(\mathrm{COOH}=$ $1.34 \mathrm{mmol} / \mathrm{g}$ ), $0.03 \mathrm{~g}$; NVC, $3.0 \mathrm{~g}$; toluene, $10.0 \mathrm{~mL} ; 80^{\circ} \mathrm{C}$.

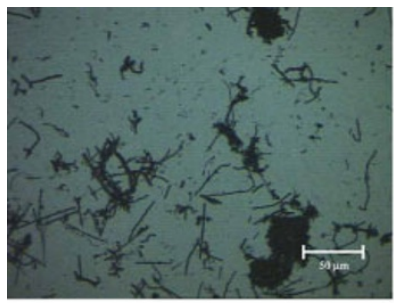

Untreated CMC

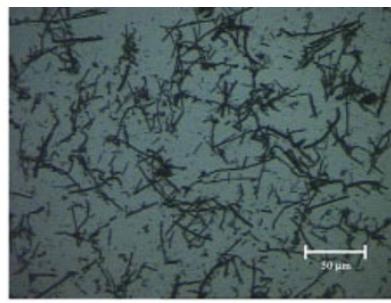

PEG-grafted CMC

Figure 7. Optical microscope aspects of untreated and PEGgrafted CMC in water. CMC content, $5.0 \mathrm{wt} \%$.

In addition, the cationic polymerization of $\mathrm{NVC}$ was also initiated at $80^{\circ} \mathrm{C}$ to give polyNVC-grafted CMC. It was found that polyNVC grafting increased with progress of the polymerization and reached to $25.1 \%$ after $10 \mathrm{~h}$ as shown in Figure 6.

These results indicate that the cationic polymerization was initiated by proton addition to monomer from carboxyl groups on CMC and the propagation proceeds from surface carboxylate anion as counter ion as shown in Scheme 2. The corresponding polymer was grafted onto the surface based on the termination reaction of growing polymer cation with carboxylate anion on the surface as shown in Scheme 2.

\section{Dispersibility of Polymer-Grafted CMC}

Figure 7 shows optical microscope aspects of untreated and PEG-grafted CMC in water. It was found that untreated CMC was entangled each other, but the entanglement was effectively destroyed by grafting of PEG onto the surface of CMC.

In addition, Figure 8 shows optical microscope aspects of surface of composite prepared from silicone rubber and polyIBVE-grafted CMC. It was found that untreated $\mathrm{CMC}$ was entangled each other in silicone rubber, but polyIBVE-grafted CMC uniformly dispersed in silicone rubber. 


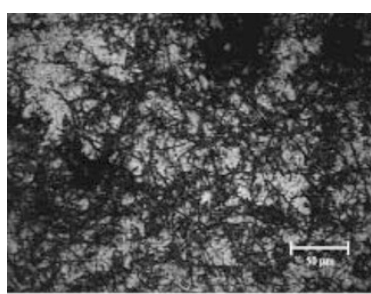

Untreated CMC

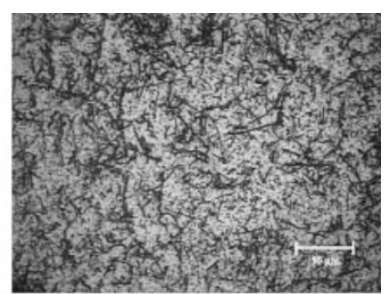

PolyIBVE-grafted CMC
Figure 8. Optical microscope aspects of untreated and IBVEgrafted CMC in silicone rubber. CMC content, $5.0 \mathrm{wt} \%$.

\section{Electric Properties of Conductive Composite Prepar-} ed from Silicone Rubber and Polymer-Grafted CMC

Figure 9 shows the response of the inductance $L$, capacitance $C$, and resistance $R$ of the silicone rubber composite prepared from silicone rubber and polyIBVE-grafted CMC against to acetone vapor. The inductance of the composite suddenly increased in acetone vapor, and returned to almost initial value when it was transferred into air. On the other hand, the capacitance and resistance of the composite decreased in acetone vapor, and returned to almost initial value when it was transferred into air. But response of resistance to acetone vapor was unstable. Inductance in air gradually increased and capacitance in air gradually decreased with repeating exposure to acetone vapor. This may be due to the fact that acetone vapor absorbed by silicone rubber hardly desorbed completely in air for $5 \mathrm{~min}$.

It was confirmed that the weight of the conductive composite immediately increased $0.13 \%$ after $1 \mathrm{~min}$ when it was transferred in acetone vapor and decreased gradually $0.10 \%$ after $5 \mathrm{~min}$ when it was transferred to dry air.

Figure 10 shows the response of the inductance of the composite prepared from PEG-grafted carbon black to acetone vapor. The inductance of the composite also increased in acetone vapor, and returned to almost initial value when it was transferred into air.

Figure 11 shows the response of the inductance of the composite prepared from untreated carbon black and $\mathrm{CMC}$ to acetone vapor. It became apparent that the inductance of the composite prepared from carbon black, which is particle and has no coil structure, hardly changed in acetone vapor. On the contrary, the inductance of the composite from CMC responded to acetone vapor, but the response was unstable.

The volume increase of the composite in acetone vapor at $25^{\circ} \mathrm{C}$ was negligibly small, but the volume increase (about 10\%) was observed in acetone vapor at $50^{\circ} \mathrm{C}$.

Based on the above results, it is considered that the expansion of CMC based on the swelling of silicone rubber by adsorption of acetone vapor causes the
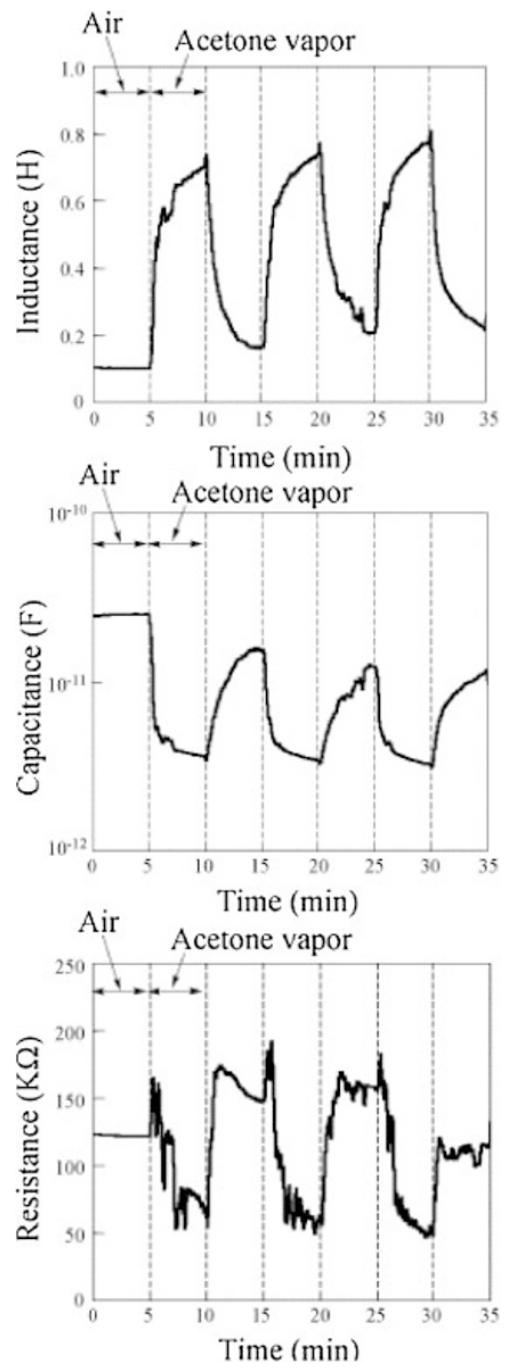

Figure 9. Response of inductance, capacitance, and resistance of the composite prepared from silicone rubber and polyIBVEgrafted CMC (grafting $=18.5 \%$ ) to acetone vapor at $25^{\circ} \mathrm{C}$.

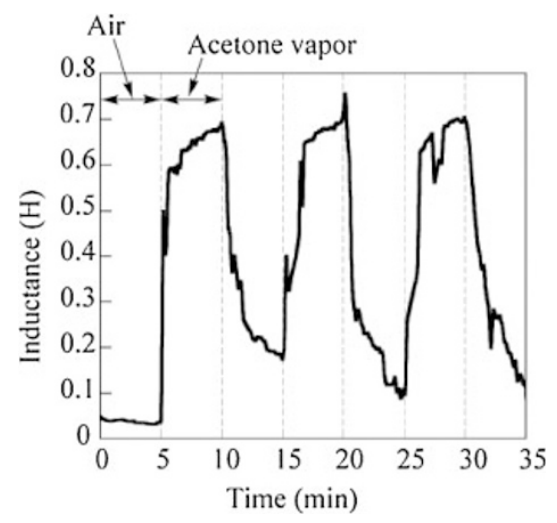

Figure 10. Response of inductance of the composite prepared from silicone rubber and PEG-grafted CMC $\left(M_{\mathrm{n}}=3400\right.$, grafting $=15.0 \%$ ) to acetone vapor at $25^{\circ} \mathrm{C}$.

changes of inductance, capacitance and resistance.

In comparison of Figure 9 with Figure 11, it was found that the increase of inductance of the composite prepared from untreated $\mathrm{CMC}$ was smaller and more 


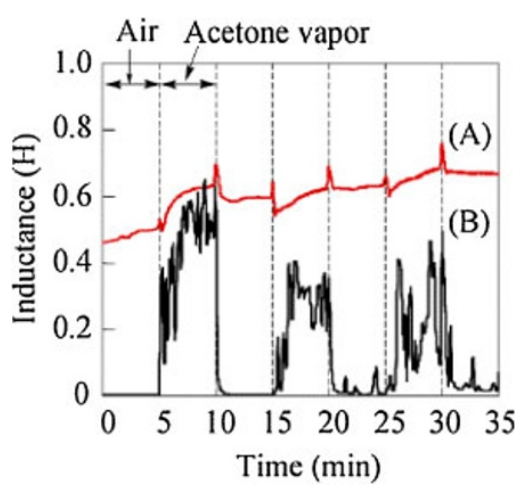

Figure 11. Response of inductance of the conductive composite prepared from (A) carbon black and (B) untreated CMC to acetone vapor at $25^{\circ} \mathrm{C}$.

unstable than those prepared from polyIBVE-grafted CMC. It seems that the uniform dispersion of CMC in silicone rubber by grafting polymer causes effective expansion and contraction of CMC in silicone rubber.

Based on the above results, the conductive composites prepared from polymer-grafted CMC and silicone rubber is a possible candidate of sensitive organic vapor sensor.

\section{CONCLUSIONS}

1. Carboxyl groups were successfully introduced on CMC surface by the treatment with nitric acid. The carboxyl group content increased with increasing reaction time and reached to $1.34 \mathrm{mmol} / \mathrm{g}$ after $10 \mathrm{~h}$.

2. PEG was grafted onto CMC surface by the reaction of carboxyl groups with terminal hydroxyl groups in the presence of DCC or $\mathrm{Sc}(\mathrm{OTf})_{3}$.

3. Carboxyl groups on CMC surface have ability to initiate the cationic polymerization of vinyl monomers, such as IBVE and NVC and the corresponding polymers were grafted onto the surface.

4. Grafting of polymers onto the surface effectively destroyed the entanglement of CMC. Polymer-grafted CMC uniformly dispersed in good solvent for grafted chain and silicone rubber.

5. The inductance, capacitance, and resistance of the conductive composite prepared from polymer-grafted $\mathrm{CMC}$ responded to acetone vapor. The inductance of the composite from polymer-grafted CMC suddenly increased in acetone vapor, and returned to initial value when it was transferred into air. The capacitance and resistance of the composite decreased in acetone vapor, and returned to initial value when it was transferred into air.

\section{REFERENCES}

1. S. Motojima, Y. Hishikawa, and H. Iwanaga, Recent. Dev. Mater. Sci., 3, 633 (2002).

2. Y. Furuya, T. Hashishin, H. Iwanaga, S. Motojima, and Y. Hishikawa, Carbon, 42, 331 (2004).

3. X. Chen, S. Yang, M. Hasegawa, K. Kawabe, and S. Motojima, Appl. Phys. Lett., 87, 054101 (2005).

4. T. Katsuno, X. Chen, S. Yang, S. Motojima, M. Homma, T. Maeno, and M. Konyo, Appl. Phys. Lett., 88, 232115 (2006).

5. H. Morita, M. Honma, T. Maeno, M. Konyou, S. Motojima, and N. Kawabe, The Society of Instrument and Control Engineers, Abstract of 22th Sensing Forum, 331 (2005).

6. N. Tsubokawa and K. Fujiki, in "Polymeric Materials Encyclopedia," J. C. Salamone, Ed., CRC Press, New York, 1996, vol. 5, p 3251.

7. N. Tsubokawa, Bull. Chem. Soc. Jpn., 75, 2115 (1992).

8. N. Tsubokawa, Polym. J., 37, 637 (2005).

9. N. Tsubokawa, H. Hamada, and Y. Sone, Polym. Plast. Technol. Eng., 28, 201 (1989).

10. G. Wei, K. Fujiki, H. Saitoh, K. Shirai, and N. Tsubokawa, Polym. J., 36, 316 (2004).

11. N. Tsubokawa, H. Maruyama, and Y. Sone, Polym. Bull., 15, 209 (1986).

12. G. Wei, K. Fujiki, H. Saitoh, K. Shirai, and N. Tsubokawa, Carbon, 42, 1923 (2004).

13. N. Tsubokawa, N. Takeda, and K. Kudoh, Nippon Kagaku Kaishi, 1264 (1980).

14. N. Tsubokawa, N. Takeda, and T. Iwasa, Polym. J., 13, 1093 (1981).

15. N. Tsubokawa, M. Hosoya, and J. Kurumada, React. Funct. Polym., 27, 75 (1995).

16. N. Tsubokawa and M. Takamura, Polym. Prepr., Jpn., 54, 564 (2005).

17. N. Tsubokawa, N. Abe, G. Wei, J. Chen, and S. Saitoh, J. Polym. Sci., Part A: Polym. Chem., 40, 1868 (2002).

18. G. Wei, S. Saitoh, H. Saitoh, K. Fujiki, T. Yamauchi, and N. Tsubokawa, Polymer, 45, 8723 (2004).

19. H. Morohashi, Y. Nishita, Y. Takahashi, K. Fujiki, T. Yamauchi, and N. Tsubokawa, Polym. J., 39, 175 (2007).

20. M. Okazaki, K. Maruyama, M. Tsuchida, and N. Tsubokawa, Polym. J., 31, 672 (1999).

21. J. Chen and N. Tsubokawa, Polym. Adv. Technol., 11, 101 (2000).

22. H. Iwata, T. Nakanoya, H. Morohashi, J. Chen, T. Yamauchi, and N. Tsubokawa, Sens. Actuators, B, 113, 875 (2006).

23. H. Morohashi, T. Nakanoya, H. Iwata, T. Yamauchi, and N. Tsubokawa, Polym. J., 38, 548 (2006).

24. D. Rivin, Rubber Chem. Technol., 36, 729 (1963). 